\title{
Fomento de la Participación del Alumnado con Herramientas Libres de Trabajo Colaborativo Web 2.0
}

\author{
Manuel Palomo, Antonio García, Francisco Palomo e Inmaculada Medina \\ Universidad de Cádiz, Departamento de Lenguajes y Sistemas Informáticos, Escuela Superior de \\ Ingeniería, C/ Chile, 1. 11002 Cádiz-España (e-mail: manuel.palomo@uca.es, \\ antonio.garciadominguez@uca.es, francisco.palomo@uca.es, inmaculada.medina@uca.es)
}

Recibido Jun. 23, 2010; Aceptado Ago. 31, 2010; Versión final recibida Sep. 23, 2010

\begin{abstract}
Resumen
Este artículo presenta tres experiencias llevadas a cabo en la Universidad de Cádiz (España) durante los cursos 2008-2009 y 2009-2010. En ellas se emplean herramientas Web 2.0 para facilitar el trabajo en grupo de los alumnos, tanto en clase como fuera de ella. Se persigue este objetivo en dos frentes: creación de documentos con tecnologías wiki y desarrollo colaborativo de proyectos de software. Como consecuencia, todo el trabajo que se realiza está disponible públicamente y se usan herramientas automáticas para su medición, lo que facilita proporcionar retroalimentación a los alumnos y hace más transparente la evaluación. Los resultados muestran que estas tecnologías facilitan la detección de problemas en el aprendizaje y en el trabajo interno de los grupos. Además, los alumnos valoran positivamente este tipo de iniciativas en las que son protagonistas. Sin embargo, ese mayor grado de participación hace necesario el uso de herramientas automáticas para su seguimiento.
\end{abstract}

Palabras clave: ingeniería informática, trabajo colaborativo, wiki, software libre

\section{Promoting Participation of Students using Web 2.0 Collaborative Open Source Tools}

\begin{abstract}
This paper presents the results of three experiences developed in the University of Cádiz (Spain) during the courses 2008-2009 and 2009-2010. Web 2.0 tools to promote collaborative work both inside and outside the classroom are employed. Efforts are concentrated along two lines: writing documentation with wiki technology, and collaborative software development. All the work done by the students is publicly available, and automated tools are used for measuring it, which facilitate the feedback to students and make the evaluation process more transparent. Results show that due to these technologies, individual learning problems and problems within workgroups can be easily detected by the instructor. Besides, students feel comfortable with an initiative that turns them into the main actors in the class. Nevertheless, this higher degree of participation makes it necessary the use of automated tools to control the progress of the students.
\end{abstract}

Keywords: computer science, collaborative work, wiki, open source software 


\section{INTRODUCCIÓN}

En los últimos años se observa cómo las tecnologías Web 2.0 (blogs, wikis, redes sociales, etc.) han propiciado que los usuarios de la red pasen de ser meros consumidores de información publicada por otras entidades a autores de contenidos con un público potencial enorme. Cabe destacar el avance que se ha producido en algunas herramientas de creación de contenidos digitales como MediaWiki, la tecnología que emplea el proyecto Wikipedia, cuyo éxito ha llevado recientemente a Microsoft a abandonar la comercialización de su famosa enciclopedia Encarta (20minutos, 2010), y en los sistemas colaborativos de desarrollo de software, que hacen factible el desarrollo de software por parte de personas que, las más de las veces, ni siquiera se conocen físicamente y que trabajan de manera distribuida (Raymond, 2001). Este artículo presenta los resultados de tres experiencias llevadas a cabo en la Universidad de Cádiz (UCA), España, durante los cursos 2008-2009 y 2009-2010. Estas iniciativas están enmarcadas en tres Proyectos de Innovación Educativa Universitaria cuyo principal objetivo es fomentar el trabajo en equipo, convirtiendo a los alumnos en los verdaderos protagonistas de las asignaturas, tanto en clase como en su trabajo fuera de ella. En concreto, este objetivo se desarrolla en dos frentes: creación colaborativa de documentación técnica con tecnologías wiki y desarrollo colaborativo de proyectos software con un sistema de control de versiones.

Las asignaturas implicadas son «Programación Funcional» y «Razonamiento Automático» de Ingeniería Informática, y «Diseño de Videojuegos» de Ingeniería Técnica en Informática de Sistemas. En las dos primeras se han desarrollado dos wikis, WikiHaskell y WikiRA, respectivamente, en los que los alumnos crean materiales complementarios a los proporcionados en clase. En la tercera se lleva a cabo el desarrollo colaborativo de software con control de versiones. En ellas, todo el trabajo que se realiza está disponible públicamente. De este modo se apela a la responsabilidad del alumno, porque no está en laboratorio, sino en un wiki o repositorio de código disponible en Internet que "hablará de él", tanto para bien (si crean documentación útil o código de calidad), como para mal (si comenten faltas de ortografía en el wiki o producen código con errores). Aunque existen iniciativas similares (Chao y Parker, 2007) tanto en wikis (VillanuevaGarcía, 2009) como en desarrollo colaborativo de software (Milentijevic et al., 2008), el grado de automatización del seguimiento del alumno es limitado (Dodero et al., 2009). En las experiencias aquí presentadas, se usan herramientas automáticas (algunas disponibles previamente, y otra creada al efecto) para el seguimiento del alumno, lo que facilita la retroalimentación y hace más transparente la evaluación. Otro aspecto a destacar es el empleo exclusivo de tecnologías libres, no sólo en el aula, sino durante el trabajo individual que debe realizar cada alumno externamente. Este propósito está fundamentado no sólo en razones éticas (González et al., 2004), sino también por motivos prácticos, y se encuentra en consonancia con la declaración institucional de apoyo al software libre realizada por el Consejo de Gobierno de la UCA (UCA, 2004). Hay que aclarar que estas herramientas son de calidad igual o superior a sus equivalentes privativas (CENATIC, 2010), y su uso ha sido declarado de interés para el fomento de la competitividad del sector TIC de la Unión Europea (Ghosh et al., 2007).

Las asignaturas implicadas participan en el Campus Virtual de la UCA, que se sustenta en la plataforma libre Moodle (Palomo et al., 2006). Este facilita la difusión de materiales en formato electrónico y permite la entrega electrónica de trabajos, la realización de tutorías electrónicas y la comunicación entre todos los interesados a través de foros temáticos para la discusión de problemas, prácticas de laboratorio o cualesquiera otros aspectos relacionados. En este sentido cabe destacar la iniciativa de la UCA que permite a los alumnos solicitar en las bibliotecas de los centros ordenadores portátiles en préstamo, en condiciones similares a los libros, facilitando el acceso al Campus Virtual en cualquier momento gracias a la red inalámbrica que la universidad tiene en sus cuatro campus. Igualmente, las asignaturas participantes sufren un doble proceso de evaluación: un primer proceso que ejecuta la Unidad de Calidad de la UCA y un segundo proceso, interno, que abarca aspectos más específicos de la materia propia del curso y que no suelen ser tratados por la Unidad de Calidad, que persigue objetivos más generales. Además, a lo largo del curso se recoge información puntual sobre la evolución y el rendimiento de los alumnos. El resto del artículo está organizado del siguiente modo: en la segunda sección se presenta la experiencia de creación colaborativa de documentación técnica con tecnologías wiki. En la tercera sección se discuten los proyectos de desarrollo colaborativo de software. Por último, se ofrecen conclusiones y se incluyen agradecimientos y referencias. 


\section{CREACIÓN COLABORATIVA CON TECNOLOGÍAS WIKI}

La tecnología libre MediaWiki es la usada actualmente por la mayoría de los wikis libres. Está basada en PHP, y permite conexiones con bases de datos MySQL y PostgreSQL. Entre los wikis que la usan se incluyen Wikipedia y sus proyectos hermanos (como Wiktionary, Wikibooks, Wikisource, etc.), para los que fue concebida originalmente. Esta tecnología fue creada por Lee Daniel Crocker y es mantenida en la actualidad por empleados de la Fundación Wikimedia y algunos voluntarios. Dada la difusión de este proyecto, existen cada vez más extensiones que añaden nuevas funciones al sistema, como gestión de acceso de usuarios (MediaWiki, 2010) o control de vandalismos (Rodríguez, 2010). MediaWiki incluye varios niveles de usuarios, estando reservadas ciertas acciones al grupo de administradores (como borrar páginas o bloquear a otros usuarios). Pero la potencia del software radica en que cualquier persona con pocos conocimientos informáticos pueda modificar el contenido fácilmente. A esto se añade la sencillez para crear, enlazar y clasificar páginas, facilitando la navegación por los contenidos. Estos no sólo incluyen texto, sino también imágenes, sonidos e incluso vídeos.

\section{Análisis de wikis con StatMediaWiki}

Para facilitar el seguimiento y evaluación del trabajo del alumnado en los wikis de estas experiencias se ha creado una herramienta de análisis estadístico de wikis MediaWiki: StatMediaWiki. Este sistema está disponible para descarga gratuita bajo licencia libre (Rodríguez et al., 2010). Similar a aplicaciones como StatSVN, permite observar la actividad de los usuarios, los progresos en los contenidos que se generan y recopila métricas para ver quienes aportan al wiki y en qué cantidad. El análisis generado muestra, primero, un resumen global con el número de páginas, ediciones totales, número de usuarios y de ficheros subidos. En la figura 1 se detalla la evolución de, en concreto, WikiHaskell a lo largo del tiempo con unas gráficas que permiten ver el número de bytes añadidos y la actividad general según la hora del día y el día de la semana.

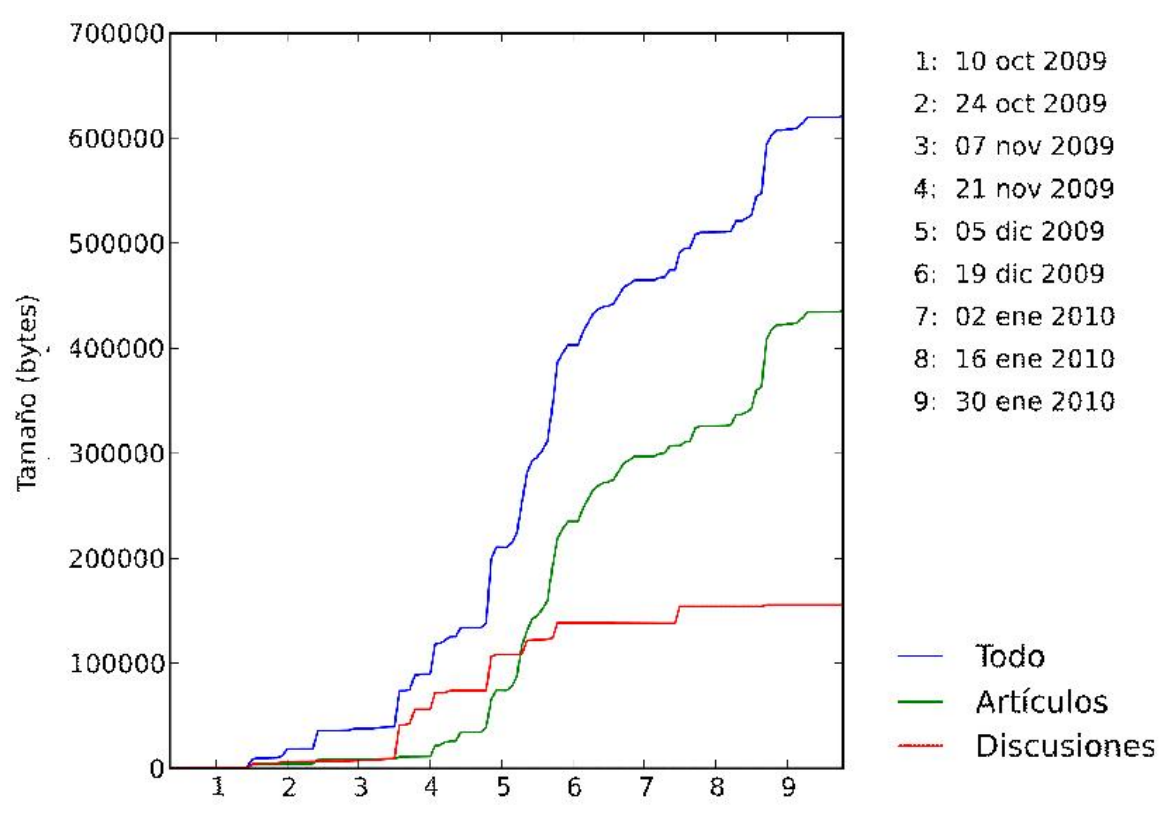

Fig. 1: Evolución del tamaño de WikiHaskell (StatMediaWiki, 2010)

La tabla 1 proporciona un listado de los usuarios que han trabajado en el wiki ordenados por modificaciones realizadas, con el número de bytes añadidos y la cantidad de ficheros subidos. Un ranking de páginas más editadas permite ver qué contenidos han sufrido más modificaciones y revisiones. También, es posible observar qué palabras clave se han utilizado en mayor medida durante las ediciones al wiki (nube de tags). Además, el análisis que proporciona la herramienta no sólo ofrece una visión general, sino que permite ver, usuario a usuario, cuál ha sido el progreso de este a lo largo del tiempo, el contenido añadido, las horas y días de la semana donde ha trabajo más, las páginas donde ha realizado más modificaciones, y una galería con las imágenes aportadas al wiki. 
Tabla 1: Clasificación de usuarios de WikiHaskell (StatMediaWiki, 2010)

\begin{tabular}{|l|l|c|c|c|c|c|}
\hline$\#$ & Usuario & $\begin{array}{c}\text { Ediciones } \\
\text { Totales }\end{array}$ & $\begin{array}{c}\text { Ediciones en } \\
\text { artículos }\end{array}$ & Bytes añadidos & $\begin{array}{c}\text { Bytes añadidos en } \\
\text { artículos }\end{array}$ & Ficheros \\
\hline 1 & Alumno1 & $175(11.78 \%)$ & $87(7.75 \%)$ & $209.882(30.31 \%)$ & $26.017(5.51 \%)$ & 0 \\
\hline 2 & Alumno2 & $129(8.68 \%)$ & $54(4.81 \%)$ & $12.668(1.83 \%)$ & $8.390(1.78 \%)$ & 0 \\
\hline 3 & Alumno3 & $75(5.05 \%)$ & $49(4.37 \%)$ & $39.309(5.68 \%)$ & $32.997(6.99 \%)$ & 1 \\
\hline 4 & Alumno4 & $63(4.24 \%)$ & $54(4.81 \%)$ & $28.478(4.11 \%)$ & $27.219(5.76 \%)$ & 2 \\
\hline 5 & Alumn05 & $62(4.17 \%)$ & $61(5.44 \%)$ & $15.185(2.19 \%)$ & $14.851(3.14 \%)$ & 0 \\
\hline 6 & Alumno6 & $54(3.63 \%)$ & $27(2.41 \%)$ & $31.382(4.53 \%)$ & $26.789(5.67 \%)$ & 0 \\
\hline 7 & Alumno7 & $51(3.43 \%)$ & $41(3.65 \%)$ & $19.058(2.75 \%)$ & $18.683(3.96 \%)$ & 9 \\
\hline 8 & Alumno8 & $50(3.36 \%)$ & $49(4.37 \%)$ & $23.145(3.34 \%)$ & $23.109(4.89 \%)$ & 0 \\
\hline 9 & Alumno9 & $49(3.30 \%)$ & $47(4.19 \%)$ & $5.614(0.81 \%)$ & $5.525(1.17 \%)$ & 0 \\
\hline 10 & Alumno10 & $38(2.56 \%)$ & $37(3.30 \%)$ & $11.854(1.71 \%)$ & $11.292(2.39 \%)$ & 0 \\
\hline & Subtotal & $746(50.20 \%)$ & $506(45.10 \%)$ & $396.575(57.27 \%)$ & $194.872(41.27 \%)$ & 12 \\
\hline
\end{tabular}

StatMediaWiki se ha empleado en el análisis de WikiHaskell y WikiRA, como apoyo para la evaluación de los alumnos, obteniendo resultados bastante satisfactorios que serán concretados en las próximas secciones.

\section{WikiHaskell}

El desarrollo del proyecto WikiHaskell se encuadra dentro del Proyecto de Innovación Educativa titulado «Empleo de tecnologías colaborativas web 2.0 para fomentar el trabajo en equipo del alumnado», aprobada con código PIE-101 en la convocatoria del año 2009 de Proyectos de Innovación Educativa Universitaria para el Personal Docente e Investigador de la UCA (UCA, 2009).

\section{WikiHaskell: Objetivo}

El principal objetivo de este proyecto es incorporar al aula la creación de conocimiento libre, haciendo que los alumnos se conviertan en los verdaderos protagonistas de la asignatura, tanto en clase como fuera de ella. Para ello se crea, en grupos de tres alumnos, materiales complementarios a los proporcionados en clase para «Programación Funcional», asignatura optativa de cuarto/quinto curso de la titulación de Ingeniero en Informática de la UCA. Esta asignatura introduce un paradigma de programación novedoso para el alumno (la programación funcional), que ha estudiado previamente el paradigma imperativo y el orientado a objetos. Supone aprender una nueva forma de enfocar y resolver problemas que produce soluciones generales, elegantes y más fáciles de verificar. Pensamos que mediante las actividades implicadas en el proyecto presentado en este artículo se facilita la adquisición de esta nueva forma de enfocar y resolver problemas (Gibbs y Simpson, 2005).

También, pensamos que se favorece la adquisición de determinadas habilidades, como las de trabajo en grupo, autoaprendizaje, expresión escrita o análisis crítico (Wheeler et al., 2008). Los alumnos aprenden las dificultades que conlleva la redacción de documentación técnica de calidad y el profesor cuenta con una herramienta valiosa para observar el progreso de los alumnos y, en particular, qué materias son las que presentan una mayor dificultad para el alumno y dónde se producen confusiones o lagunas en los conceptos y técnicas que estos deben dominar.

\section{WikiHaskell: Desarrollo}

Durante el desarrollo de la asignatura, el alumno sigue una evaluación continua a través de la realización y superación de las siguientes tareas y actividades de obligado cumplimiento: 
1. Pruebas individuales presenciales de control escritas. Suponen el $30 \%$ de la nota.

2. Resolución de ejercicios: desarrollo de funciones (ejercicios de programación mediante ordenador y escritos sobre papel). Representan el $25 \%$ de la nota final.

3. Realización de trabajos: desarrollo de materiales complementarios a los proporcionados en clase en WikiHaskell y elaboración de críticas y resúmenes sobre artículos y conferencias. En total suman el $35 \%$ de la nota final: $25 \%$ para el desarrollo de materiales complementarios en WikiHaskell.

4. Generación de dudas y selección de las más útiles y frecuentes (DUF). La generación de este DUF también se hace dentro de WikiHaskell y supone un $10 \%$ de la nota final.

Para el desarrollo de WikiHaskell, se dividió al alumnado en grupos de tres seleccionados de forma aleatoria para conseguir simular, dentro de nuestras limitaciones, lo que ocurre en la vida laboral: hay que trabajar en un equipo cuyos integrantes normalmente no puedes elegir. Cada grupo selecciona, entre las bibliotecas disponibles en el lenguaje Haskell, la que va a usar para generar los materiales complementarios. Durante este curso se ha documentado: GHC6-Network, la biblioteca gráfica Gtk2Hs, la biblioteca astar, HOMMAGE, la biblioteca IO, la biblioteca libSDL, el paquete gnuplot, la biblioteca de empaquetamiento Cabal, las pruebas unitarias para Haskell, la biblioteca HPDF, la biblioteca HDBC, la biblioteca C Math y RSA-Haskell. Además, cada grupo realiza presentaciones periódicas en clase en las que explican el estado actual de su trabajo con WikiHaskell, sus últimos avances y los problemas encontrados. Esto permite conocer la opinión de los alumnos sobre las técnicas de trabajo utilizadas y su progreso en el proyecto. Es imprescindible para la evaluación positiva que los integrantes del grupo vayan rotando, de manera que cada presentación periódica la haga un componente distinto del grupo.

Para fomentar el interés de los alumno se pusieron como requisitos indispensables:

- Asistencia sistemática a las sesiones que traten sobre WikiHaskell.

- Trabajo individual y en grupo. Cada alumno debe hacer de forma individual y en grupo aportaciones en WikiHaskell, tanto en el DUF como en los materiales complementarios.

- Seguimiento continuo y planificado. Se valora positivamente que las aportaciones a WikiHaskell se hagan de forma continua. Con esto se fomenta que el alumnado trabaje de forma continua y no lo deje todo para el final.

- Evaluación por pares. Se exige el seguimiento y evaluación de los trabajos realizados por otros compañeros.

- Realización y entrega de las actividades en las fechas fijadas.

En cuanto a los criterios de evaluación se tienen en cuenta:

- Adecuación a los principios del paradigma de la programación funcional.

- Adecuación a estándares y a la especificaciones.

- Eficiencia en la ejecución de los programas realizados.

- Organización, claridad, elegancia y corrección de las soluciones presentadas.

- Participación e implicación.

- Corrección ortográfica y gramatical.

\section{WikiHaskell: Resultados}

Este proyecto comenzó su andadura en el primer cuatrimestre del curso 2009-2010. Los resultados han sido muy positivos. A pesar de tener un número significativo de alumnos (en concreto 46), todos han rendido a un nivel bastante alto, especialmente, en cuanto a su trabajo con WikiHaskell. De hecho, de los 46, 40 han aprobado, ha habido 4 no presentados y sólo dos suspensos. Aplicando StatMediaWiki las cifras obtenidas nos indican que se han realizado 1.487 modificaciones con un total de 629.581 bytes, de las cuales 1.123 se han producido en 44 páginas (el resto son principalmente en páginas de discusión sobre bibliotecas de Haskell). Porcentualmente esto nos dice que cada alumno ha hecho una media de algo más de 32 aportaciones al wiki con un total de 15.124 bytes por alumno. Creemos que la sensación de seguimiento que tenían los alumnos al poder acceder a las estadísticas de StatMediaWiki en tiempo real y su valoración en la nota final de la asignatura ayudaron a que se involucraran en el wiki, evitando crear un ambiente de indiferencia como en otras experiencias similares (Cole, 2009). 
También es curioso observar que el conjunto de los 10 alumnos que más aportaciones han realizado (que son algo más del $20 \%$ de la clase) suman alrededor del $50 \%$ de las aportaciones al wiki, lo que demuestra que por lo general la participación ha sido bastante distribuida (StatMediaWiki, 2010). Además, gracias a las gráficas generadas por StatMediaWiki, se han podido identificar cinco perfiles de alumnos:

- Perfil continuo: lo consideramos el perfil óptimo. El alumno va haciendo aportaciones de forma continua durante todo el desarrollo del trabajo. Sólo 3 de los alumnos han cumplido este perfil.

- Perfil en escalón: este es también un perfil bueno, el alumno va haciendo aportaciones de forma continua aunque algo intermitente. De todos los alumnos 16 han seguido este perfil.

- Perfil pico al principio: este es el perfil del abandono, ya que lo siguen alumnos que sólo realizaron aportaciones al principio pero que después abandonaron el trabajo y la asignatura. Sólo 4 alumnos siguen este perfil.

- Perfil pico a mitad: junto con el perfil en escalón, este es el que más han seguido los alumnos, 17 en total. En este, la mayor parte del trabajo la realizan a mitad del periodo de desarrollo.

- Perfil pico al final: este es el perfil del alumno que deja el trabajo para última hora. Sólo 6 de los alumnos cumplieron este perfil.

Además, también se han obtenido datos sobre las horas y días de la semana en el que más trabajan. A grandes rasgos, se ha trabajado más los días entre semana, los fines de semana apenas se han hecho aportaciones. En cuanto a las horas, es bastante variable aunque suelen trabajar más por las mañanas (lógico ya que las clases presenciales de la titulación son en horario de tarde). Dado que es una actividad evaluable en clase que cuenta el 25 por ciento de la nota final, el wiki sólo permite que los alumnos de la asignatura puedan hacer modificaciones. Sin embargo, el contenido sí es públicamente accesible desde (Medina et al., 2010). En una encuesta anónima que se realizó al alumnado tras terminar la asignatura, y que respondieron 24 alumnos, se obtuvieron los resultados que se pueden ver en la tabla 2 donde el rango de respuesta era de 1 (poco) a 5 (mucho).

Tabla 2: Encuesta anónima realizada al alumnado sobre WikiHaskell

\begin{tabular}{|l|c|}
\hline Pregunta & Media \\
\hline Opinión sobre el uso del wiki libre con acceso público en docencia & 4,50 \\
\hline Desarrollo del trabajo en el wiki (grupos de tres alumnos, organización y revisiones) & 3,83 \\
\hline Dificultad de usar el wiki & 2,54 \\
\hline Peso del trabajo en el wiki en la nota final de la asignatura & 3,67 \\
\hline Valoración general de la asignatura & 4,13 \\
\hline
\end{tabular}

\section{WikiRA}

El desarrollo del proyecto WikiRA se encuadra, al igual que el proyecto WikiHaskell, dentro del Proyecto de Innovación Educativa «Empleo de tecnologías colaborativas web 2.0 para fomentar el trabajo en equipo del alumnado», aunque también está dentro del Proyecto de Innovación Educativa titulado «Desarrollo y control de flujos de trabajo para asignaturas de Informativa empleando LAMS», aprobada con código PIE-28 en la convocatoria del año 2009 de Proyectos de Innovación Educativa Universitaria para el Personal Docente e Investigador de la UCA (UCA, 2009).

\section{WikiRA: Objetivo}

El principal objetivo de este proyecto es, al igual que en el caso de WikiHaskell, incorporar al aula la creación de conocimiento libre, haciendo que los alumnos se conviertan en los verdaderos protagonistas de la asignatura, tanto en clase como fuera de ella. Para ello se crea materiales complementarios a los proporcionados en clase para «Razonamiento Automático», asignatura optativa de cuarto/quinto curso de la titulación de Ingeniero en Informática de la UCA. Sin embargo, a diferencia del experimento realizado en WikiHaskell, en este caso no se han formado grupos de tres, sino que en WikiRA se ha trabajado de forma individual y sin hacer un seguimiento muy estrecho de los progresos que se iban realizando durante el desarrollo de la asignatura. El 
contenido de WikiRA es públicamente accesible desde (Palomo et al., 2010). Hay que tener en cuenta que las asignaturas optativas se enfrentan al dilema de garantizar un aprendizaje en profundidad de la materia propia de estudio sin que ello llegue a amenazar su propia existencia. Si la carga de trabajo implicada se antoja elevada, los contenidos desarrollados se perciben complejos o la evaluación resulta estricta, los alumnos optan invariablemente por la deserción, lo que en el contexto actual de recortes presupuestarios suele implicar la desaparición de la asignatura.

Nótese el empleo deliberado de los verbos antojar, percibir y resultar. El objeto común es el alumno que, en más ocasiones de las debidas, no desea convertirse en estudiante, si existen alternativas. La dificultad intrínseca de la asignatura importa sólo hasta un cierto punto; lo que realmente importa es la percepción que se tenga de ella. Sería prolijo discutir las razones por las que existen dichas alternativas, pero en el caso de las optativas resultan sobradamente conocidos qué criterios primarán en la elección de la matrícula y cómo los reglamentos en vigor en muchas universidades españolas no hacen sino favorecer la continuidad de esta situación, con la consiguiente degradación de la calidad de las asignaturas optativas respecto a las de carácter obligatorio. No obstante, afortunadamente también existen verdaderos estudiantes que están dispuestos a asumir el reto, si la asignatura les ofrece un extra. Algo que haga llevadero, o que al menos disimule, el esfuerzo adicional que habrán de dedicar a su superación. Este extra es la motivación y, a continuación, expondremos como con acciones como esta de convertir al alumno en verdadero protagonista de la asignatura se puede conseguir.

\section{WikiRA: Desarrollo}

Durante el desarrollo de la asignatura, el alumno sigue una evaluación continua a través de la realización y superación de una serie de tareas y actividades de obligado cumplimiento. El programa de la asignatura no invita precisamente a la matriculación, pero es el que requiere una asignatura de estas características, sea o no del gusto del alumno: automatización del razonamiento lógico, comprobación de modelos, verificación, etc. Todas estas unidades poseen sólidos prerrequisitos de lógica y de técnicas de programación avanzadas. Es necesario emplear diversos formalismos lógicos (lógicas de primer orden, temporales, etc.), así como varios lenguajes de programación y herramientas especializadas. En particular, los alumnos han de emplear herramientas libres de análisis léxico (Flex) y sintáctico (Bison) para crear traductores escritos en lenguaje $\mathrm{C}++$ que conviertan entre diferentes representaciones, como por ejemplo, para pasar fórmulas lógicas escritas de manera natural con símbolos Unicode a notación Lisp. También aprenden a manejar un comprobador de satisfacibilidad (SATO) (Zhang, 2010) y, de hecho, han de programar su propia versión del algoritmo DPLL en un subconjunto funcional puro de Common Lisp. Para trabajar con lógicas de primer orden se ha empleado el demostrador de teoremas Otter (o su sucesor, Prover9) (McCune, 2010) y para la comprobación de modelos mediante lógica temporal, SAL (Rushby, 2010). La lógica computacional ACL2 posee su propia herramienta, homónima (Kaufmann et al., 1995).

Todas las herramientas empleadas son de libre distribución, suministrándose los enlaces o incluso el propio software, a través del campus virtual. Se dispone de un sistema de evaluación continua con cuatro hitos o entregas formales, entre los que está el trabajo colaborativo de WikiRA. La calificación final de la asignatura es la media de las calificaciones obtenidas en cada una de las entregas. Para el desarrollo de WikiRA no se divide al alumnado en grupos, sino que se plantea como una actividad colaborativa a desarrollar durante todo el curso, de manera que recoja contribuciones individuales de cada uno de los alumnos pero de forma integrada (Palomo et al., 2009). El objetivo es que se sientan partícipes, aunque sea en pequeña medida, del proceso de creación de materiales de aprendizaje.

\section{WikiRA: Resultados}

Este proyecto comenzó su andadura en el segundo cuatrimestre del curso 2009-2010. Los resultados han sido positivos pero no tanto como con WikiHaskell. Pensamos que las principales razones para esto son, por un lado, que los alumnos han trabajado de forma individual en vez de 
en grupo, y por otro, que el seguimiento realizado del wiki no ha sido tan estricto como en el caso de WikiHaskell (StatMediaWiki, 2010). En el curso actual, debido a la reducción paulatina del número de optativas y a la implantación de un plan de ciclos de optatividad (no todos los años se imparten todas las optativas), la asignatura comenzó el curso con un número inusualmente alto de matriculados, 34. De los cuales 19 han aprobado, ha habido 12 no presentados y 3 suspensos. La tabla 3 muestra el número de suspensos (trabajos entregados que no alcanzan el mínimo exigido) y de abandonos por entrega. En un par de casos excepcionales, alumnos que abandonaron una de las entregas pudieron reincorporarse con éxito a la asignatura; en general, el abandono, cuando se produce, es definitivo.

Aplicando StatMediaWiki las cifras obtenidas indican que se han realizado 473 modificaciones con un total de 247.649 bytes, de las cuales 345 se han producido en 27 páginas. Porcentualmente esto nos dice que cada alumno ha hecho una media de 14 aportaciones al wiki con un total de 7.283 bytes por alumno, aunque haciendo los cálculos sobre los 22 presentados, obtenemos una media de algo más de 21 aportaciones con un total de 11.256 bytes por alumno. También es curioso observar que el conjunto de los 10 alumnos que más aportaciones han realizado (que son casi el $30 \%$ de la clase) suman alrededor del $62 \%$ de las aportaciones al wiki, lo que demuestra que por lo general la participación ha sido bastante distribuida. Además, con las gráficas generadas por StatMediaWiki, se ha podido contrastar la diferencia de perfiles de los alumnos respecto a los que había en WikiHaskell. En concreto, en WikiRA la mayoría de los alumnos están dentro del perfil pico al final.

Tabla 3: Evolución de los suspensos y abandonos por entrega

\begin{tabular}{|c|c|c|c|}
\hline Entrega & Suspensos & Abandonos & Tasa de abandonos (\%) \\
\hline 1 & 3 & 10 & 29 \\
\hline 2 & 0 & 2 & 6 \\
\hline 3 & 1 & 0 & 0 \\
\hline 4 & 10 & 0 & 0 \\
\hline
\end{tabular}

Esto último tiene una explicación clara y es que no se llevó, deliberadamente, un seguimiento demasiado estrecho durante su desarrollo. Esto indica que aunque los alumnos estén motivados en realizar una determinada actividad, se suelen dejar ir si no tienen que ir dando cuentas de su trabajo de forma periódica. También pensamos que el hecho de que las aportaciones fueran individuales y no en grupo, hace que el alumno se sienta menos comprometido en el trabajo y, por consiguiente, que los resultados hayan sido algo peores que en WikiHaskell. Por tanto, estamos convencidos de que para que este tipo de proyectos colaborativos funcionen se deben realizar en grupo y se debe llevar a cabo un seguimiento minucioso del desarrollo (Judd, et al., 2010).

Por otro lado, al igual que con WikiHaskell, también se han obtenido datos sobre las horas y días de la semana en el que más trabajan llegando a las mismas conclusiones. A grandes rasgos, se ha trabajado más los días entre semana, los fines de semana apenas se han hecho aportaciones. En cuanto a las horas, es bastante variable aunque suelen trabajar más por las mañanas. Durante el curso, la asignatura ha sido sometida a dos procesos de evaluación. El primero, externo, ha sido llevado a cabo por la unidad de calidad de la UCA y consiste en una evaluación relativamente extensa de distintos aspectos de la asignatura a varios niveles de detalle (desde la puntualidad del profesor hasta el estado de las instalaciones), si bien no se dispone aún de sus resultados. El segundo, interno, abarca aspectos más técnicos de la asignatura, como el peso de las diferentes actividades (como el wiki cooperativo) en la nota final, la preparación y conocimientos del profesor, etc.

Esta segunda evaluación, a diferencia de la primera, incluye la posibilidad de que el alumno incluya comentarios a cada una de sus respuestas, bien sea para matizarlas, expresar una queja o proponer una mejora. Desde el punto de vista de los autores, aunque esto impide que su tratamiento se realice automáticamente, dichos comentarios son en ocasiones más valiosos que la valoración en sí. En resumen, los alumnos valoran globalmente a la asignatura con un 3,1 sobre 5 . 
Ambas evaluaciones se llevaron a cabo anónimamente, sin conocimiento previo de su fecha de realización por los alumnos y con los presentes en clase (en ambos casos, el 56\% de los matriculados, aproximadamente). En la tabla 4 pueden verse los resultados.

Tabla 4: Encuesta anónima realizada al alumnado sobre WikiRA

\begin{tabular}{|l|c|}
\hline Pregunta & Media \\
\hline Opinión sobre el uso del wiki libre con acceso público en docencia & 3,4 \\
\hline Desarrollo del trabajo en el wiki (organización y revisiones, etc.) & 3,2 \\
\hline Dificultad de usar el wiki & 2,2 \\
\hline Peso del trabajo en el wiki en la nota final de la asignatura & 3,2 \\
\hline Valoración general de la asignatura & 3,1 \\
\hline
\end{tabular}

\section{DESARROLLO COLABORATIVO DE SOFTWARE CON CONTROL DE VERSIONES}

Se denominan tecnologías de control de versiones (en inglés Control Version System) a una serie de sistemas informáticos que permiten controlar el desarrollo de software de manera distribuida. Mediante una sencilla interfaz (en consola o integrada en los entornos de desarrollo más populares) es posible que desarrolladores distribuidos por Internet puedan guardar, recuperar y mezclar diferentes versiones de archivos entre otras operaciones. Estos sistemas suelen usarse integrados en una forja de desarrollo de código. Una forja es un entorno online que proporciona una serie de herramientas de última generación que simplifican la organización de ciertas tareas de desarrollo colaborativo de software y facilitan el trabajo distribuido. Suelen incluir, además de sistemas de control de versiones, herramientas de comunicación (listas de correos y foros), gestión de tareas pendientes (con asignaciones a desarrolladores, prioridades y fechas tope), publicación de noticias con RSS, documentación, gestión de informes externos de fallos, etc. Toda esta información está disponible de manera pública en tiempo real, lo que posibilita el seguimiento constante del trabajo de los alumnos por el profesor. Apache Subversion (también conocido como SVN) es un sistema para el control de versiones que está soportado por las principales forjas de software libre. Es clave para que los distintos miembros de un grupo puedan realizar desarrollo de código de manera asíncrona y distribuida (Wang, 2009).Permite que, por ejemplo, un alumno amplíe parte de un videojuego mientras otro hace un módulo nuevo y otro corrige errores. Y después, cuando los usuarios den el visto bueno, el sistema incorpora las distintas aportaciones al repositorio de código y las redistribuye entre ellos.

\section{Herramientas de análisis: CVSAnalY y StatSVN}

La herramienta más interesante que hemos encontrado para generar estadísticas de un repositorio SVN es la utilidad libre CVSAnalY (Robles et al., 2004). Esta herramienta analiza los registros de cada una de las versiones enviadas al repositorio y produce una base de datos relacional que puede ser consultada de forma flexible para generar todo tipo de informes. Es compatible con otros sistemas de control de versiones, como CVS o Git. CVSAnalY es una herramienta extensible, pudiendo activar componentes para generar información adicional: por ejemplo, se puede obtener información de cómo evoluciona la complejidad de los programas desarrollados, los tipos de ficheros que conforman el proyecto, o la proporción en que cada desarrollador ha contribuido. Diseñar un nuevo informe sólo requiere escribir las consultas SQL necesarias para agregar la información disponible.

Otra herramienta de interés es StatSVN (Appendium, 2010). Es un sistema libre que muestra de manera gráfica gran cantidad de información sobre el trabajo realizado en un proyecto. De este modo, es un complemento interesante a CVSAnalY, por ser más rápido y visual para detectar problemas en el trabajo de los alumnos (aunque es menos detallado). Además, es interesante el seguimiento temporal que hace de las aportaciones de cada desarrollador. Podemos ver a qué horas suele realizar aportaciones cada integrante de un grupo, así como el día de la semana y del mes. Esta información, aunque no es interesante para la evaluación, creemos que permite a los alumnos reflexionar sobre su modo de trabajo, cuándo rinden más, etc. 


\section{Diseño de Videojuegos}

La asignatura «Diseño de Videojuegos» es optativa de tercer curso de Ingeniería Técnica en Informática de Sistemas de la UCA. Tiene una carga de 6 créditos LRU (4,5 créditos ECTS) y un marcado carácter multidisciplinar, pues integra conocimientos de programación, inteligencia artificial, física, modelado, etc. La asignatura se imparte desde el curso 2006-2007, pero no fue hasta el pasado curso 2008-2009 cuando se explican y usan técnicas de desarrollo colaborativo y se desarrollo el producto en una forja bajo licencia libre. La asignatura se encuadra dentro del Proyecto de Innovación Educativa titulado «Empleo de tecnologías colaborativas web 2.0 para fomentar el trabajo en equipo del alumnado», aprobado con código PIE-101 en la convocatoria del año 2009 de Proyectos de Innovación Educativa Universitaria para el Personal Docente e Investigador de la UCA (UCA, 2009).

\section{Diseño de Videojuegos: Objetivo}

La asignatura se desarrolla usando aprendizaje basado en proyectos (Álvarez et al., 2009): el primer día de clase los alumnos organizan grupos de tres que trabajarán juntos a lo largo del cuatrimestre desarrollando un videojuego en dos dimensiones con la biblioteca multimedia multiplataforma libSDL. Dicho proyecto se aloja en la Forja de Conocimiento Libre (RedIRIS, 2010) de la Comunidad RedIRIS (red española para Interconexión de los Recursos InformáticoS de las universidades y centros de investigación), con el fin de dar mayor visibilidad a dichos proyectos y utilizar las herramientas de última generación que esta incorpora.

\section{Diseño de Videojuegos: Desarrollo}

La mayoría de alumnos afirman que este trabajo es el proyecto más grande con el que se enfrentan en su formación universitaria, comprendiendo cuatro meses de trabajo de tres personas. Por ello, se insiste en clase en los aspectos de organización del trabajo: planificación del trabajo con diagramas de Gantt, generación automática de documentación con Doxygen, etc. Como además la temática de los videojuegos resulta, por lo general, de interés para los alumnos (al ser una asignatura optativa cursada voluntariamente), se cuenta con una disposición muy buena del alumnado para llevar su proyecto a buen término, registrándose muy pocos problemas internos en los grupos.

La nota de la asignatura se divide en dos partes que valen un $50 \%$ cada una: nota individual y nota de grupo. La primera es independiente para cada miembro del grupo, pero la segunda es igual para todos ellos. Así se consigue un equilibrio entre un objetivo común (el proyecto) y la aportación personal de cada miembro del equipo. La nota individual se subdivide en tres componentes: participación en clase (30\%), nota de la competición de sistemas expertos (30\%) (esta experiencia puede consultarse en (Recio-Quijano et al., 2010)) y aportación individual al proyecto (40\%). En cambio, la nota de grupo se reparte en cuatro componentes que se corresponden con cada una de las presentaciones mensuales que deben de hacer de su proyecto. La nota de cada una de ellas va aumentando a medida que es mayor el trabajo realizado: la primera presentación vale el $10 \%$ de la nota, pues incluye principalmente la planificación del proyecto con asignaciones de tareas y los recursos multimedia a usar (imágenes, sonidos, etc). La segunda cuenta el $20 \%$ de la nota, y consiste básicamente en el diseño general de videojuego y el prototipo que programan los alumnos. La tercera entrega consiste en una versión alfa con detección y tratamiento de colisiones, que vale el 30\% de la nota. Y por último, la cuarta presentación vale el $40 \%$ restante, y es una beta con inteligencia artificial simple. Como se observa, no se exige que se entregue un producto terminado y estable: el objetivo de la asignatura es aprender a diseñar e implementar un videojuego, y entendemos que con un videojuego en estado beta se aprende todo lo necesario. Consideramos que el resto de actividades (corrección de fallos, modificaciones de la arquitectura por problemas técnicos encontrados en la implementación, etc.) son más pesadas, y su alcance se escapa del trabajo exigible a un alumno en un cuatrimestre. Además, normalmente suelen deberse a imperfecciones del diseño, por lo que el hecho de reconocerlas implica que el alumno ha aprendido cuál sería el diseño correcto, así que sólo se le pide que las comentarte en la última presentación. 
Las presentaciones las hacen todos los grupos el mismo día. Esto hace que los que van más retrasados se den cuenta cuenta de que tienen que esforzarse más. Además, el uso de las herramientas automáticas que se comentan en el siguiente apartado permite ver si el grupo o el alumno en particular no ha trabajo o lo ha hecho pero se ha encontrado con dificultades. La evaluación se realiza con rúbricas que han sido publicadas y discutidas con los alumnos previamente. De esta forma se fomenta el trabajo autónomo del alumno, aplicando lecciones aprendidas en experiencias de implantación de técnicas escalables para gestionar grupos grandes de alumnos aplicando técnicas del Espacio Europeo de Educación Superior (EEES) (Estero et al., 2006).

\section{Diseño de Videojuegos: Resultados}

CVSAnalY proporciona algunos datos que reflejan el carácter multimedia de los videojuegos desarrollados en la asignatura: de los 8.749 ficheros producidos por los alumnos entre los cursos 2008-2009 y $2009-2010$, el $24,25 \%$ se ha dedicado a código, mientras que un $34,37 \%$ se dedica a las imágenes y audio de los juegos, y un 22,48\% a documentar el propio código, usando Doxygen. El 18,89\% restante se dedica a ficheros de apoyo, empaquetado y soporte en general. La actividad de los alumnos ha sido continua en ambas ediciones, desarrollándose en los períodos de marzo a junio, principalmente, con una ligera aceleración antes de las hitos intermedios de presentación de los proyectos, como puede verse, por ejemplo, en la figura 2 de 2009.

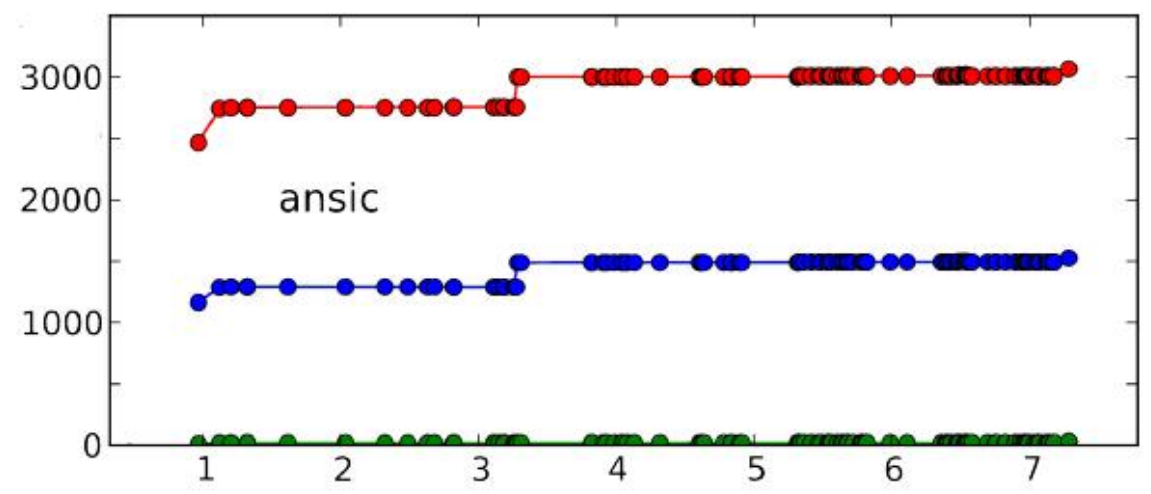

1: 28 mar 2009

2: 11 abr 2009

3: 25 abr 2009

4: 09 may 2009

5: 23 may 2009

6: 06 jun 2009

7: 20 jun 2009

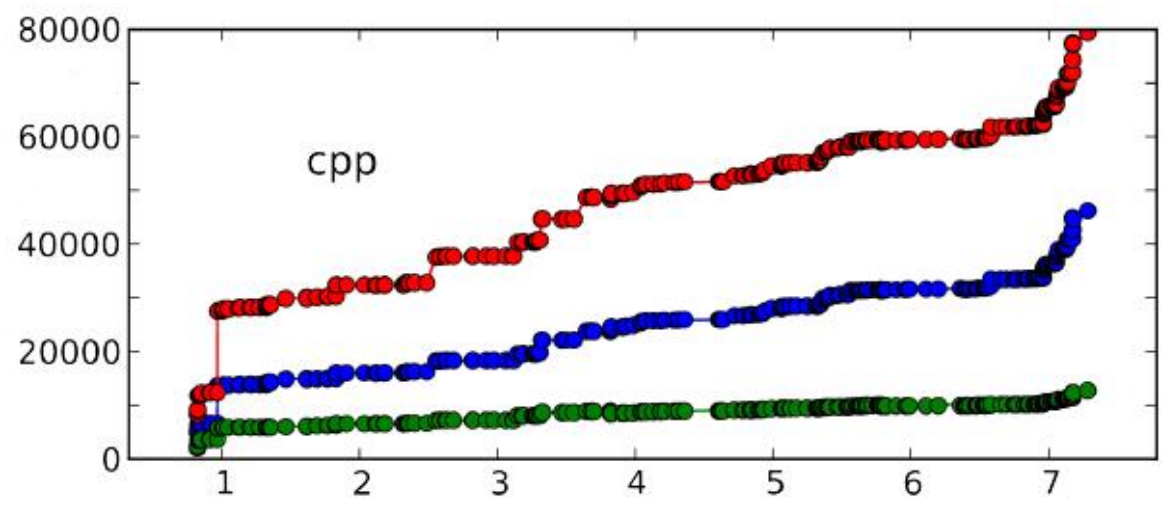

$\because$ SLOC

$\because \mathrm{CLOC}$

$\because$ LOC

Fig. 2: Crecimiento del código en el año 2009

Sin embargo, algunos alumnos han continuado sus contribuciones tras del final de la asignatura, pasando a ser un proyecto personal más que un simple trabajo de clase, como es el caso de "Granny's Bloodbath" (figura 3, siguiente página). En eella se distingue entre las líneas de código propiamente dicho (SLOC), las líneas dedicadas a comentar el código (CLOC) y los totales (LOC).

Otro aspecto interesante revelado por los análisis realizados es la división de labor creada dentro de cada uno de los proyectos. En general, cada proyecto ha tenido un programador jefe que es el principal contribuidor del código, mientras que los otros miembros se han dedicado a crear y reunir materiales gráficos y sonoros, asistir en la documentación o realizar pruebas y corregir errores. En 
algunos casos, como el del proyecto "Robinson 2.0", el rol de programador jefe se ha trasladado de un desarrollador (arld101) a otro (theomega), como puede verse en la figura 4 de la siguiente página.

Consideramos que, en líneas generales, el trabajo realizado por los alumnos es considerable para ser una asignatura cuatrimestral optativa. Destacamos la media entre los cursos 2008-2009 y 2009-2010 de 47 aportaciones al proyecto con un total de más de 35 ficheros creados por alumno. También es interesante ver cómo gracias a la generación automática de documentación las 177.884 líneas de código de todos los proyectos del curso 2008-2009 han generado casi 110.000 líneas de documentación (la diferencia entre el total de líneas en bruto y las de código).

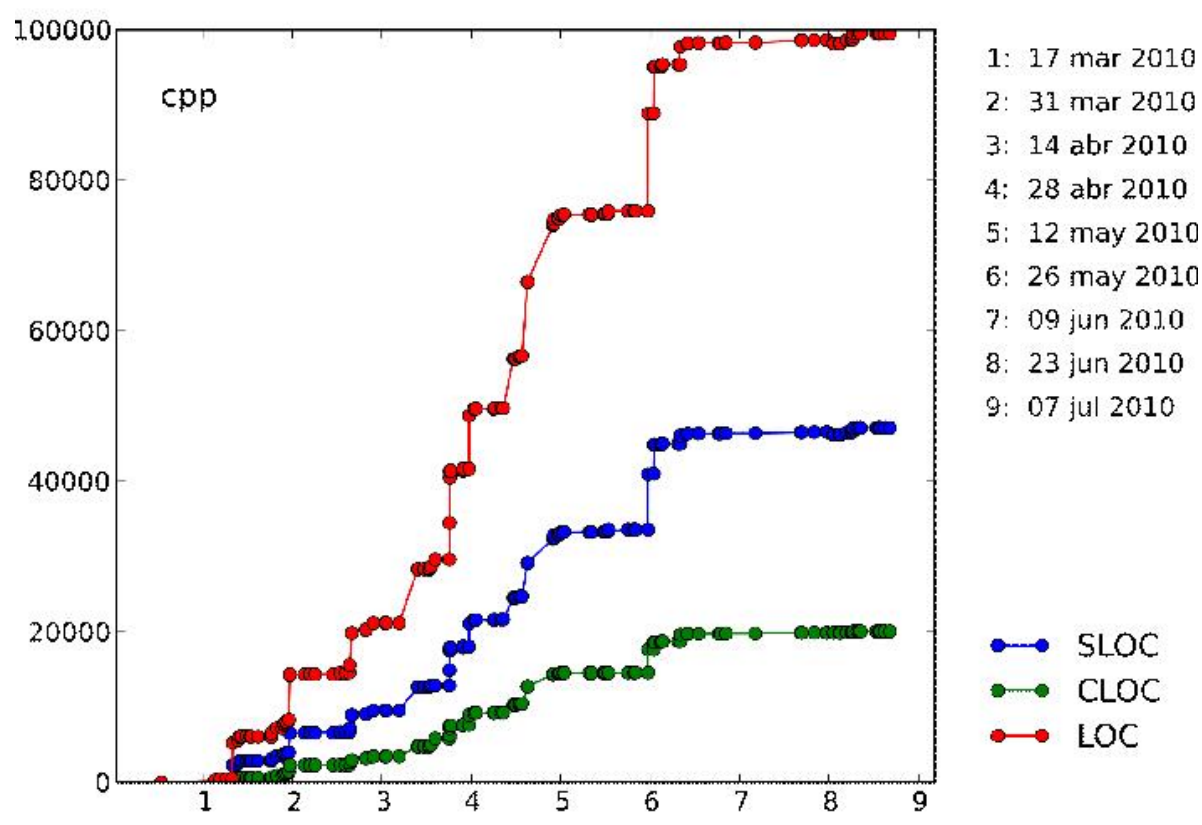

Fig. 3: Crecimiento del código en el proyecto "Granny's Bloodbath"

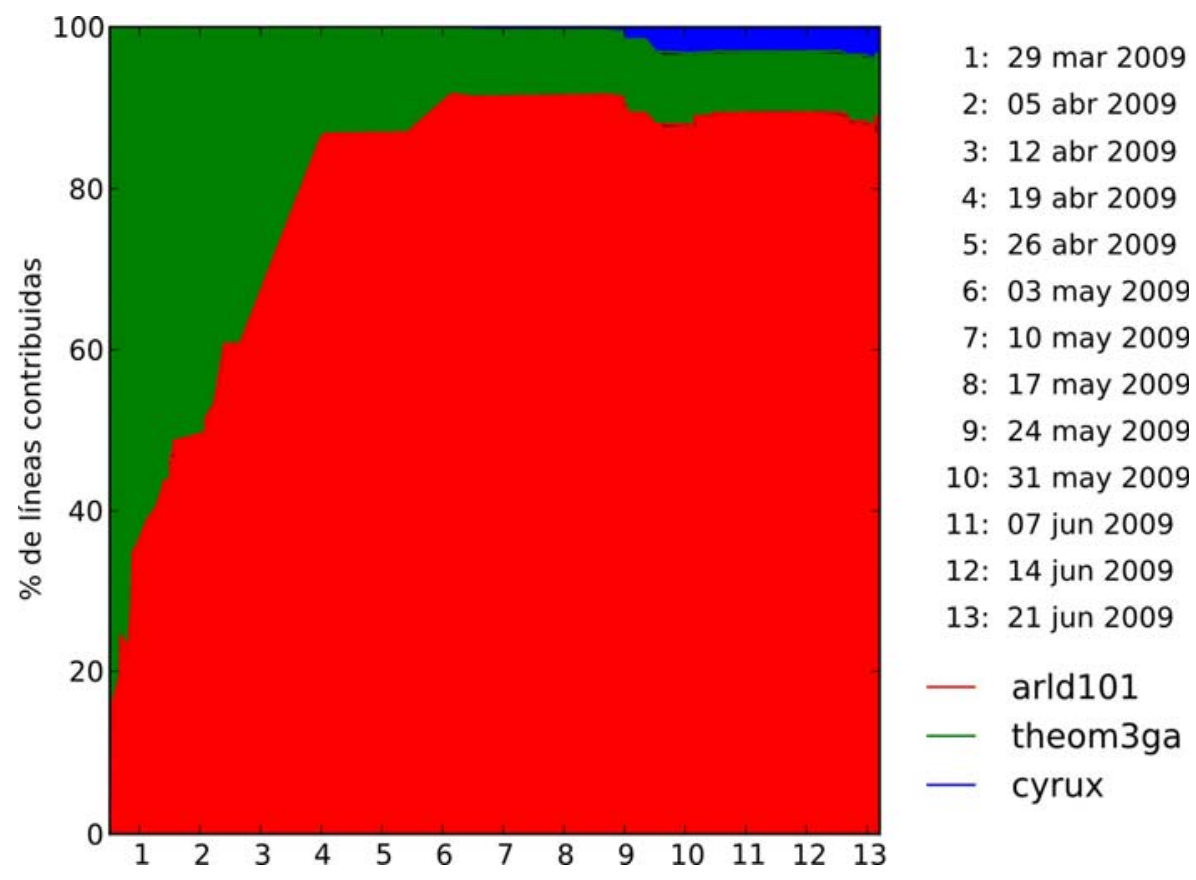

Fig. 4: Evolución de la proporción de cambios por usuario en el proyecto "Robinson 2.0" 
StatSVN permite clasificar a los alumnos por perfiles. Observando por ejemplo, alumnos que prefieren trabajar de noche (algunos tienen casi el 40\% de las aportaciones de madrugada), mientras que otros lo hacen casi por igual durante todo el día. En cuanto a los días de la semana algunos grupos aprovechan el tiempo de clase para trabajar y sus aportaciones se centran en los días de clase, a diferencia de otros que centran su trabajo en el fin de semana. Sin embargo, también hay comportamientos generalizados, cómo la concentración de aportaciones los días previos a la fecha de las presentaciones intermedias. Los datos académicos de la asignatura han sido muy buenos en los dos cursos (11 notables, 27 sobresalientes y 2 abandonos). Los datos de la encuesta realizada se muestran en la tabla 5.

Tabla 5: Encuesta anónima realizada al alumnado sobre Diseño de Videojuegos

\begin{tabular}{|l|c|c|}
\hline Pregunta & $\begin{array}{c}\text { Media } \\
2008 / 9\end{array}$ & $\begin{array}{c}\text { Media } \\
2009 / 10\end{array}$ \\
\hline Desarrollo de la asignatura en proyectos en grupos de tres alumnos & 4,52 & 4,09 \\
\hline Valore el uso de la Forja de RedIRIS & 4,41 & 4,91 \\
\hline Valore el uso de Subversion & 4,44 & 4,91 \\
\hline Evaluación de la asignatura & 4,48 & 4,55 \\
\hline Valoración general de la asignatura & 4,74 & 4,82 \\
\hline
\end{tabular}

\section{CONCLUSIONES}

Como conclusiones podemos destacar en primer lugar, que existen a día de hoy herramientas libres de calidad que proporcionan una cobertura total a las necesidades de asignaturas de Ingeniería Informática. Además estos sistemas facilitan enormemente el trabajo del profesor para seguir cada proyecto y alumno de manera individual en tiempo real, una de las claves para la exitosa implantación de los nuevos títulos adaptados a la Declaración de Bolonia.

Nuestra experiencia muestra que estas tecnologías hacen más fácil detectar problemas en el aprendizaje de alumnos, en el trabajo interno de grupos, etc. Gracias al uso de herramientas automáticas como StatMediaWiki o CVSAnalY se evitan muchas tareas repetitivas y propensas a errores a la vez que se "arroja" luz sobre el trabajo realizado por los alumnos permitiendo evaluarlos de manera sencilla y transparente. Gracias a ellos hemos podido identificar varios perfiles de alumnos a lo largo de la asignatura. Cruzando esta información con su rendimiento académico permitirá en próximos cursos detectar los alumnos proclives a abandonar la asignatura y facilitará centrar en ellos nuestros esfuerzos. Igualmente, también se obtienen otros datos de interés como los días de la semana y hora del día en que más trabajan, la distribución del trabajo por alumno, etc.

Por otro lado, las encuestas muestran que los alumnos ven con muy buenos ojos su participación en este tipo de iniciativas en las que son protagonistas. Creemos que usando tecnologías que les resulten cómodas y cercanas, y estableciendo un sistema de trabajo que siendo flexible les obligue a rendir cuentas, su implicación, satisfacción y rendimiento académico puede ser muy alto. También queremos destacar la importancia para la comunidad hispana de WikiHaskell de disponer de documentación libre de calidad en español gracias a este tipo de experiencias que proporcionan una adecuada recompensa a la participación en el wiki, algo absolutamente necesario (Ebner et al., 2008).

Como trabajo futuro también podemos destacar que para el próximo curso la asignatura «Administración de Sistemas Operativos» (obligatoria de tercer curso de Ingeniería Técnica en Informática de Sistemas) incorporará desarrollo de documentación en wiki con rúbricas similar a (Trentin, 2009). Actualmente ya cuenta con un wiki, WikiUNIX (Sales et al., 2010). Pero su edición no es obligatoria para los alumnos ni se valora en la nota. Todavía no está claro el método de trabajo, aunque se está valorando hacer una aportación mixta, en la que los alumnos creen individualmente una página de un programa, y en grupo una página de un servicio o problema del entorno de la asignatura que se solucione con los programas que han trabajado individualmente. 


\section{AGRADECIMIENTOS}

Este trabajo ha sido financiado por los Proyectos de Innovación Educativa Universitaria del Personal Docente e Investigador siguientes: «Desarrollo y control de flujos de trabajo para asignaturas de Informativa empleando LAMS» (PIE-28), «Posibilidades de la Web 2.0 y de las producciones multimedia en la formación del alumnado y del profesorado» (PIE-38) y «Empleo de tecnologías colaborativas Web 2.0 para fomentar el trabajo en equipo del alumnado» (PIE-101), pertenecientes a la Convocatoria de Proyectos de Innovación Educativa Universitaria de la Universidad de Cádiz (Vicerrectorado de Tecnologías de la Información e Innovación Docente).

\section{REFERENCIAS}

20minutos, La Encarta sucumbe ante la Wikipedia, http://www.20minutos.es/noticia/460388/0/ wikipedia/encarta/enciclopedia/. Acceso: 16 de julio (2010)

Álvarez, A., Palomo, M. y Rodríguez, J. R., Experiencias en la aplicación de técnicas y herramientas de desarrollo colaborativo de software en una asignatura basada en proyectos, XVII Congreso de Innovación Educativa en las Enseñanzas Técnicas, Valencia, España, 15 a 18 de septiembre (2009).

Appendium. Página principal del proyecto StatSVN. http://www.statsvn.org. Acceso: 16 de julio (2010).

CENATIC (Centro Nacional de Referencia de Aplicación de las Tecnologías de la Información y la Comunicación TIC basadas en fuentes abiertas), Informe sobre el Panorama Internacional del Software de Fuentes Abiertas (2010).

Chao, J.T. Y Parker, K.R., Wiki as a teaching tool, Interdisciplinary Journal of Knowledge and Learning Objects, 3: 57-72 (2007).

Cole, M., Using wiki technology to support student engagement: Lessons from the trenches, Computers \& Education, 52(1): 141-146 (2009).

Dodero, J. M., Rodríguez, G. e Ibarra, M. S.. Análisis de las contribuciones a un wiki para la evaluación web de competencias, Actas de la Conferencia Conjunta Iberoamericana sobre Tecnologías de Aprendizaje, Mérida, México, 6 a 10 de julio (2009).

Ebner, M., Kickmeier-Rust, M. y Holzinger, A., Utilizing wiki-systems in higher education classes: A chance for universal access? Universal Access in the Information Society, 7: 199-207 (2008).

Estero, A., Domínguez, J. J. y Palomo, M., Hacia el EEES en una asignatura masificada. En Actas de las I Jornadas Nacionales de Intercambio de Experiencias Piloto de Implantación de Metodologías ECTS, Badajoz, España, 13 a 15 de septiembre (2006).

Ghosh, R.A. y otros, Economic impact of open source software on innovation and the competitiveness of the information and communication technologies (ICT) sector in the EU. Informe técnico para la Comisión Europea (2007).

Gibbs, G. y Simpson, C., Does your assessment support your students' learning?, Learning and Teaching in Higher Education, 1:3-31 (2005).

González, J. M., Matellán, M., de las Heras, P. y Robles, G., Sobre software libre: compilación de ensayos sobre software libre. Dykinson, Madrid, España (2004).

Judd, T,. Kennedy, G., y Cropper, S., Using wikis for collaborative learning: Assessing collaboration through contribution, Australasian Journal of Educational Technology, 26(3): 341-354 (2010).

Kaufmann, M., Moore, J S. y Manolios, P., Computer-Aided Reasoning: An Approach. Kluwer Academic Publishers, Boston, EE.UU. (1995). 
McCune, W., Prover9, University of New Mexico. http://www.prover9.org. Acceso: 16 de julio (2010)

MediaWiki Community, Mediawiki extensions. http://www.mediawiki.org/wiki/Category:Extensions/es. Acceso: 16 de julio (2010).

Medina, I. y otros. WikiHaskell. http://softwarelibre.uca.es/wikihaskell. Acceso: 16 de julio (2010).

Milentijevic, I., Ciric, V. y Vojinovic, O., Version control in project-based learning. Computers \& Education, 50(4):1331-1338 (2008).

Palomo, F. y otros. WikiRA. http://softwarelibre.uca.es/wikira. Acceso: 16 de julio (2010).

Palomo, M., Medina, I. y Palomo, F., Empleo de plataformas de aprendizaje electrónico para el fomento de la participación activa del alumnado, Actas de las I Jornadas de Innovación Educativa de la Escuela Politécnica Superior de Zamora, Zamora, España, 20 al 22 de junio (2006).

Palomo, M., Medina I., Rodríguez E. J. y Sales, N., Tecnologías wiki y conocimiento abierto en la universidad. Proceedings of the 5th Free Software World Conference, Cáceres, España, 1 a 3 de diciembre (2009).

Raymond, E. S., The Cathedral and the Bazaar: Musings on Linux and Open Source by an Accidental Revolutionary, O'Reilly, Sebastopol, EE.UU. (2001).

Recio-Quijano, P., Sales-Montes, N., García-Domínguez, A. y Palomo-Duarte, M., Collaboration and competitiveness in project-based learning, Proceedings of the ACM SIGCSE Methods and Cases in Computing Education Workshop (MCCE), 8-14, Cádiz, España, 30 de junio (2010).

RedIRIS, Red Española de Investigación. Forja libre de RedIRIS. http://forja.rediris.es. Acceso: 16 de julio (2010).

Robles, G., Koch, S. y González-Barahona, J. M., Remote analysis and measurement of libre software systems by means of the cvsanaly tool. Proceedings of the 2nd ICSE Workshop on Remote Analysis and Measurement of Software Systems (RAMSS), 51-55, Edinburgh, United Kingdom (2004).

Rodríguez, E. J., AVBOT: Detección y corrección de vandalismos en Wikipedia. Novática, 203:5153 (2010).

Rodríguez, E. J. y otros, Statmediawiki. http://statmediawiki.forja.rediris.es/. Acceso: 16 de julio (2010).

Rushby, J., Symbolic analysis laboratory (SAL), Sri International. http://sal.csl.sri.com. Acceso: 16 de julio (2010).

Sales, N. y otros, WikiUnix. http://softwarelibre.uca.es/wikiunix. Acceso: 16 de julio (2010).

StatMediaWiki, Estadísticas de StatMediaWiki sobre varios wikis. http://osl.uca.es/statmediawiki/. Acceso: 16 de julio (2010).

Trentin, G., Using a wiki to evaluate individual contribution to a collaborative learning project. Journal of Computer Assisted Learning, 25: 43-55 (2009).

UCA (Universidad de Cádiz), Convocatoria de proyectos de innovación educativa universitaria del Vicerrectorado de Tecnologías de la Información e Innovación Docente (2009).

UCA (Universidad de Cádiz), Acuerdo del Consejo de Gobierno de 15 de marzo de 2004, por la que se aprueba la adhesión a la declaración institucional de apoyo al uso de Software Libre en la Universidad de Cádiz. Boletín Oficial de la Universidad de Cádiz, (9):78-79, (2004).

Villanueva-García, A., Uso de wikis en ingeniería informática. Revista de Docencia Universitaria, Monográfico V - WIKI y educación superior en España (II parte) (2009). 
Wang, Q., Design and evaluation of a collaborative learning environment. Computers \& Education, 53(4): 1138-1146 (2009).

Wheeler, S., Yeomans, P. y Wheeler, D., The good, the bad and the wiki: Evaluating studentgenerated content for collaborative learning, British Journal of Educational Technology, 39(6): 987995 (2008).

Zhang, H., Sato: A solver for propositional satisfiability, University of lowa. http://www.cs.uiowa.edu/ hzhang/sato. Acceso: 16 de julio (2010). 